\title{
COMPROMETIMENTO PULMONAR NA LEPTOSPIROSE
}

\author{
Jorge Eduardo Manhães de Carvalho, Edson dos Santos Marchiori, João \\ Batista Guedes e Silva, Bernardino Alves de Souza Netto, Walter Tavares e \\ Aloysio Veiga de Paula*
}

\begin{abstract}
Em 23 pacientes com leptospirose apresentando comprometimento pulmonar, internados no Hospital Universitário Antônio Pedro da UFF, Niterói, hemoptise e hemoptóicos foram observados em $21,7 \%$ e 30,4\%, respectivamente. Gasometria arterial revelou hipoxemia e hipocapnia na maioria dos casos. Radiografia de tórax em 15 pacientesmostrou comprometimento alveolar em $60 \%$, comprometimento intersticial-reticular em 6\%, padrão misto (alveolar e intersticial) em $20 \%$ e ausência de alterações radiológicas em $14 \%$. A necrópsia de 13 pacientes mostrou edema, congestão e hemorragia nos pulmões em $100 \%$ dos casos. A hemorragia foi focal em $46 \%$ e difusa em $54 \%$ dos casos. Houve formação de membrana hialina em $30 \%$ e trombos de fibrina em $46 \%$ dos pulmóes estudados, o que estabelece o diagnóstico da coagulação intravascular disseminada e a ocorrência da sindrome de angústia respiratória na leptospirose.
\end{abstract}

Palavras-chaves:Leptospirose. Pulmão. Pneumonia. SARA. Coagulação intravascular.

A leptospirose é doença multissistêmica, de gravidade variável, onde o comprometimento de diversos órgãos contribui para sua elevada letalidade. É primariamente infecção de animais silvestres e domésticos. O homem ocasionalmente é acometido ao entrar em contato direto ou indireto (via âgua ou solo contaminado) com esses animais, principalmente com a urina de roedores infectados 113 .

A forma clássica da leptospirose, com grave acometimento renal, icterícia, hemorragias e intenso quadro febril, foi primeiro descrita por Adolf Weil, em $1886^{19} 23$. Somente em 1914, Inada e Ido, no Japão, identificaram o espiroqueta causador daquele quadro mórbido ${ }^{15}$. Doença endêmica em nosso país, teve seu estudo acrescido a partir da década de 1960, quando epidemias da enfermidade foram descritas em diferentes partes do Brasil.

A infecção é assintomática na maioria dos pacientes mas, eventualmente, manifesta-se por

\section{* In memorian.}

Trabalho realizado no Hospital Universitário Antônio Pedro da Universidade Federal Fluminense, Niterói, RJ.

Endereço para correspondência: Dr. Jorge Eduardo Manhães de Carvalho. Serviço de Pneumologia, Hospital Universitário Antônio Pedro/UFF. Rua Marquês do Paraná 303, 24030-000 Miterói, RJ.

Recebido para publicação em 12/11/90. um quadro clínico característico, de início agudo, no qual febre, mialgias, icterícia rubínica e congestão conjuntival são manifestações marcantes 12131423 . O comprometimento de diferentes órgãos pelo parasita e/ou sua(s) toxina(s), conduz ao óbito cerca de 5 a $10 \%$ dos pacientes, principalmente como resultado da agressão renal, miocárdica e vascular ${ }^{12} 24$.

O pulmão é um dos órgãos comprometidos na vigência da infecção. Neste trabalho, apresentamos os resultados de um estudo sobre as alterações pulmonares observadas em pacientes com leptospirose acompanhados no Hospital Universitário Antônio Pedro da Universidade Federal Fluminense (HUAPUFF).

\section{MATERIAL E MÉTODOS}

No período de janeiro de 1980 a abril de 1988 foram internados 136 pacientes com leptospirose no HUAP-UFF, sendo observado o comprometimento pulmonar em 23 deles, selecionados para este estudo. O diagnóstico de leptospirose foi estabelecido pelo quadro clínico característico, dados epidemiológicos indicativos da infecção (contato com água poluída), achados laboratoriais próprios da enfermidade: hemograma com leucocitose e desvio para esquerda, retenção azotada e soroaglutinação microscópica 
Carvalho JEM, Marchiori ES, Silva JBG, Souza Netto BA, Tavares W, Paula AV. Comprometimento pulmonar na leptospirose. Revista da Sociedade Brasileira de Medicina Tropical 25:21-30, jan-mar, 1992.

positiva para leptospirose. Em todos os pacientes foram pesquisados a história clínica, exame físico, evolução do quadro clínico, além dos exames complementares de rotina. Dentre estes, os de maior importância para o nosso trabalho foram a radiologia de tórax em PA e perfil, gasometria arterial e soroaglutinação microscópica para leptospirose. Nos pacientes que faleceram foi realizado o exame anatomopatológico macroscópico e microscópico.

Dos 23 pacientes estudados quanto às alterações pulmonares, dez foram selecionados com base nas alterações do exame radiológico do tórax; os 13 restantes foram pacientes que evoluíram para o óbito e cujo estudo anatomopatológico evidenciou alterações pulmonares. Nestes 23 pacientes a idade variou de 21 a 67 anos, com média de 40 anos. $O$ sexo masculino predominou (18 pacientes). Quanto à profissão, o predomínio foi de "biscateiro", entre os homens, edoméstica, entre as mulheres. Dezesseis pacientes evoluíram para o óbito, sendo realizada a necrópsia em 13; sete pacientes sobreviveram. $O$ período de incubação da doença variou de sete a 30 . dias, com média de duas semanas (Tabela 1). A principal forma de contaminação se deu no exercício da profissão, embora tenha existido referências sobre limpeza de valas e alagamento de residências ou ruas, devido a chuvas torrenciais. Foi observado, também, que esses locais estavam infestados de ratos. Onze pacientes foram submetidos à diálise peritoneal, dos quais sete evoluíram para o óbito.

O diagnóstico sorológico foi efetuado em 14

Tabela 1 - Identificação e evolução de 23 casos de leptospirose com alteraçães pulmonares.

\begin{tabular}{|c|c|c|c|c|c|c|c|c|}
\hline \multirow[b]{2}{*}{ Casos } & \multirow[b]{2}{*}{ Ident. } & \multirow[b]{2}{*}{ Idade } & \multirow[b]{2}{*}{ Sexo } & \multirow[b]{2}{*}{ Cor* } & \multirow[b]{2}{*}{ Profissão } & \multicolumn{2}{|c|}{ Dias de doença } & \multirow[b]{2}{*}{ Evolução } \\
\hline & & & & & & $\begin{array}{l}\text { antes da } \\
\text { internação }\end{array}$ & internado & \\
\hline 01 & D.B & 37 & M & $\mathrm{Pa}$ & Pintor de automóvel & 5 & 2 & óbito \\
\hline 02 & M.A.L.S. & 35 & $\mathrm{~F}$ & $\mathrm{~Pa}$ & Doméstica & 10 & 4 & óbito \\
\hline 03 & C.S.B. & 59 & F & $\mathrm{B}$ & Doméstica & 10 & 2 & óbito \\
\hline 04 & A.V.G. & 30 & M & B & Auxiliar de escritório & 7 & 1 & óbito \\
\hline 05 & J.F. & 49 & $\mathbf{M}$ & B & Não identificada & 4 & 12 & óbito \\
\hline 06 & S.G.S. & 36 & $\mathbf{M}$ & B & "Biscateiro" & 5 & 4 & óbito \\
\hline 07 & J.B.M. & 28 & M & B & Pedreiro & 5 & 17 & curado \\
\hline 08 & C.G.A. & 21 & $\mathbf{M}$ & B & Pedreiro & 7 & 19 & curado \\
\hline 09 & A.A.M. & 66 & M & B & Vendedor ambulante & 15 & 20 & óbito \\
\hline 10 & A.R.A. & 57 & $\mathbf{M}$ & B & "Biscateiro" & 7 & 4 & óbito \\
\hline 11 & A.G. & 40 & M & $\mathrm{Pt}$ & Ajudante de caminhão & 5 & 4 & óbito \\
\hline 12 & G.C.O. & 29 & M & $\mathrm{Pt}$ & Gari & $30^{* *}$ & 36 & curado \\
\hline 13 & W.C.L. & 46 & $\mathbf{M}$ & $\mathrm{Pa}$ & Não identificada & 8 & 30 & curado \\
\hline 14 & J.C.S. & 28 & M & B & Gari & 3 & 3 & óbito \\
\hline 15 & P.R.B. & 25 & $\mathbf{M}$ & $\mathrm{Pa}$ & "Biscateiro" & 7 & 2 & óbito \\
\hline 16 & D.J.F.F. & 58 & M & $\mathrm{Pa}$ & Decorador de pedra & 5 & 31 & óbito \\
\hline 17 & M.S.C.S. & 22 & M & $\mathrm{Pt}$ & Serralheiro & 6 & 7 & curado \\
\hline 18 & E.C.L. & 36 & $\mathrm{~F}$ & B & Doméstica & 6 & 15 & curado \\
\hline 19 & R.O.S. & 24 & $F$ & $\mathrm{Pt}$ & Doméstica & 5 & 5 & óbito \\
\hline 20 & J.B.F. & 60 & $M$ & B & Motorista & 5 & 3 & óbito \\
\hline 21 & L.F.D. & 28 & $\mathrm{~F}$ & $\mathrm{~Pa}$ & Doméstica & 7 & 2 & óbito \\
\hline 22 & A.F.S. & 67 & M & B & "Biscateiro" & 7 & 21 & curado \\
\hline 23 & J.A.O. & 40 & $\mathbf{M}$ & B & Cozinheiro & 5 & 3 & óbito \\
\hline
\end{tabular}

* Pa-pardo; B-branco; Pt-preto

** Paciente com quadro clínico gripal 30 dias antes da internação com melhora discreta após alguns dias e em seguida surgimento de quadro clínico com mialgias, icterícia e febre. 
Carvalho JEM, Marchiori ES, Silva JBG, Souza Netto BA, Tavares W, Paula AV. Comprometimento pulmonar na leptospirose. Revista da Sociedade Brasileira de Medicina Tropical 25:21-30, jan-mar, 1992.

pacientes, sendo positivo em 13 e negativo em um, o qual estava ainda no período septicêmico. Em três casos, a sorologia foi negativa inicialmente e se positivou no período de sete a 10 dias (Tabela 2). Os exames sorológicos realizados em quatro dos pacientes (números 7, 12, 13 e 20 da Tabela 2) revelaram inicialmente positividade para diferentes serovares. Estas amostras ao serem examinadas em

Tabela 2 - Soroaglutinação efetuada em 14 pacientes portadores de leptospirose com alterações pulmonares.

\begin{tabular}{|c|c|c|c|}
\hline Número do caso & Dias da doença & Serovar & Título \\
\hline 6 & 9 & javanica & $1 / 1.600$ \\
\hline \multirow[t]{4}{*}{7} & 11 & - & Negativo \\
\hline & 18 & javanica & $1 / 200$ \\
\hline & & canícola & $1 / 200$ \\
\hline & & icterohaemorrhagiae & $1 / 800$ \\
\hline 8 & 9 & icterohaemorrhagiae & $1 / 800$ \\
\hline 9 & 10 & icterohaemorrhagiae & $1 / 800$ \\
\hline 10 & 9 & javanica & $1 / 1.600$ \\
\hline \multirow[t]{3}{*}{12} & 43 & - & Negativo \\
\hline & 52 & canícola & $1 / 200$ \\
\hline & & icterohaemorrhagiae & $1 / 400$ \\
\hline \multirow[t]{3}{*}{13} & 40 & patoc & $1 / 400$ \\
\hline & 40 & javanica & $1 / 200$ \\
\hline & 40 & bataviae & $1 / 1.600$ \\
\hline 16 & 8 & javanica & $1 / 3.200$ \\
\hline 17 & 9 & icterohaemorrhagiae & $1 / 800$ \\
\hline \multirow[t]{2}{*}{18} & 8 & - & Negativo \\
\hline & 15 & icterohaemorrhagiae & $1 / 1.600$ \\
\hline \multirow[t]{2}{*}{20} & 7 & autumnalis & $1 / 200$ \\
\hline & 7 & andamana & $1 / 1.600$ \\
\hline 21 & 8 & icterohaemorrhagiae & $1 / 400$ \\
\hline 22 & 14 & icterohaemorrhagiae & $1 / 800$ \\
\hline 23 & 7 & - & Negativo \\
\hline
\end{tabular}


Carvalho JEM, Marchiori ES, Silva JBG, Souza Netto BA, Tavares W, Paula AV. Comprometimento pulmonar na leptospirose. Revista da Sociedade Brasileira de Medicina Tropical 25:21-30, jan-mar, 1992.

maiores diluições mostraram títulos mais elevados para somente um serovar, configurando a existência de reação cruzada na titulação primitiva. Os serovares mais encontrados foram icterohaemorrhagiae (oito pacientes) e javanica (cinco pacientes). Não se obteve isolamento de leptospiras em meios de cultura, nem o microrganismo foi evidenciado em material de necrópsia.

\section{RESULTADOS}

As manifestações clínicas pulmonares foram observadas em 20 pacientes, estando ausentes em três. Dispnéia e estertores crepitantes foram as alterações mais encontradas, seguidas de tosse, hemoptóicos, estertores bolhosos, hemoptise e, menos freqüentemente, cianose, taquipnéia e atrito pleural (Tabela 3).

A radiologia do tórax, realizada em 15 dos 23 pacientes, mostrou que o padrão radiológico mais encontrado foi o de comprometimento alveolar ( $60 \%$ dos casos), sendo localizado em $6 \%$, multifocal em $27 \%$ e difuso em $27 \%$. O padrão misto (alveolar e intersticial) foi observado em $20 \%$ dos pacientes e opadrão de comprometimento intersticial reticular em $6 \%$ (Figuras 1 e 2). Em $14 \%$ dos casos não foram evidenciadas alterações no exame radiológico do tórax. Foi considerado padrão de comprometimento alveolar multifocal o comprometimento plurilobar, preservando, porém, pelo menos um dos lobos (Figuras 1 e 2).

A gasometria arterial realizada em 10 pacientes respirando ar atmosférico, no período de internação, evidenciou hipoxemia em $60 \%$ e hipocapnia em $90 \%$.

Nos 13 casos submetidos à necrópsia, macroscopicamente os pulmões pesaram individualmente de $300 \mathrm{~g}$ a $1450 \mathrm{~g}$, com média de $846 \mathrm{~g}$ para o pulmão direito e de $750 \mathrm{~g}$ para o pulmão esquerdo. A maioria (70\% dos casos) exibia pleura lisa, branca, transparente, tendo os pulmões superfície vermelho-acastanhada, áreas vinhosas difusas e crepitação diminuída à palpação (Figura 3). Em três pacientes havia petéquias subpleurais. Em dois casos foi constatado derrame pleural bilateral, sendo que, em um deles, a coleção líquida era de aspecto hemorrágico. Um paciente apresentava área sugestiva de infarto pulmonar.
Tabela 3 -Manifestaçôes clínicas pulmonares de 23 casos de leptospirose.

\begin{tabular}{lcc}
\hline Manifestações & $N^{0}$ de casos & $\%$ \\
\hline Estertores crepitantes & 10 & 43,5 \\
Dispnéia & 7 & 34,7 \\
Tosse & 7 & 30,4 \\
Hemoptóicos & 6 & 26,0 \\
Estertores bolhosos & 5 & 21,7 \\
Hemoptise & 4 & 17,4 \\
Roncos & 4 & 17,4 \\
Sibilos & 2 & 8,7 \\
Expectoração amarelada & 2 & 8,7 \\
Cianose & 2 & 8,7 \\
Taquipnéia & 1 & 4,3 \\
Atrito pleural & &
\end{tabular}

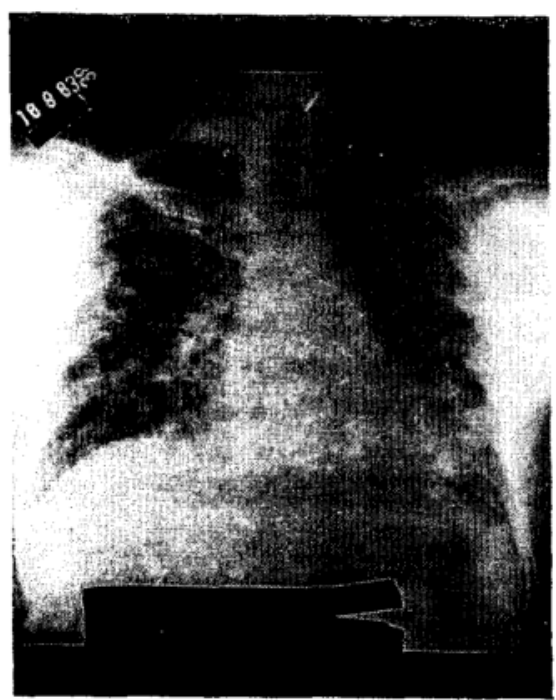

Figura 1-Telerradiografia de tórax em P.A. evidenciando condensaçóes alveolares não homogêneas nos pulmões (Padrão Alveolar Difuso). 
Carvalho JEM, Marchiori ES, Silva JBG, Souza Netto BAS, Tavares W, Paula AV. Comprometimento pulmonar na leptospirose. Revista da Sociedade Brasileira de Medicina Tropical 25:21-30, jan-mar, 1992.

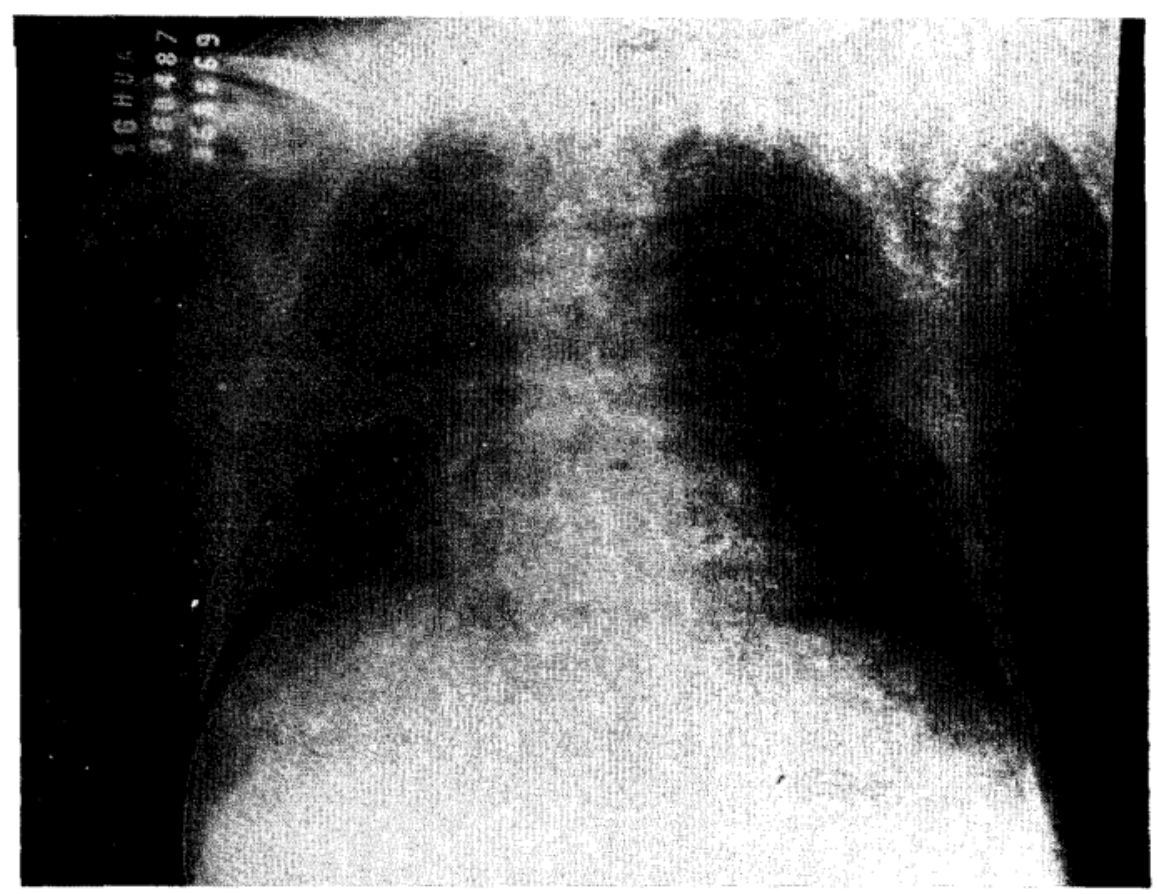

Figura 2 - Telerradiografia do tórax em P.A. evidenciando intersticial reticular difuso nos pulmões. (Padrão Intersticial Reticular).

Histologicamente, evidenciaram-se edema, congestão e hemorragia nos pulmões em $100 \%$ dos casos, sendo que a hemorragia foi focal em 6 casos (46\%) e difusa em 7 casos (54\%) (Figura 4). Apareceram, ainda, trombos de fibrina em 6 casos (46\%), membrana hialina em 4 casos $(30 \%)$, infiltrado mononuclear em 3 casos (23\%), broncopneumonia em $23 \%$, pleuris fibrinoso em $16 \%$ e pneumonite urêmica em um caso. Em oito dos 13 pacientes necropsiados $(62 \%)$ foi constatada coagulação intravascular, sendo que, nos pulmões de seis deles, havia trombos de fibrina. Em três destes seis pacientes verificou-se concomitantemente a presença de membranas hialinas (Figuras 3, 4, 5 e 6).

\section{DISCUSSÃO}

As alterações pulmonares, que representam parte do envolvimento orgânico da leptospirose, geralmente surgem precocemente, 24 a 72 horas após o seu início, ou, então, depois de alguns dias, coincidindo com o começo da fase imune ${ }^{12} 1421$.

Neste estudo de 23 pacientes com leptospirose apresentando alterações pulmonares, verificamos que, funcionalmente, a maioria apresentou hiperventilação alveolar, com hipocapnia no sangue

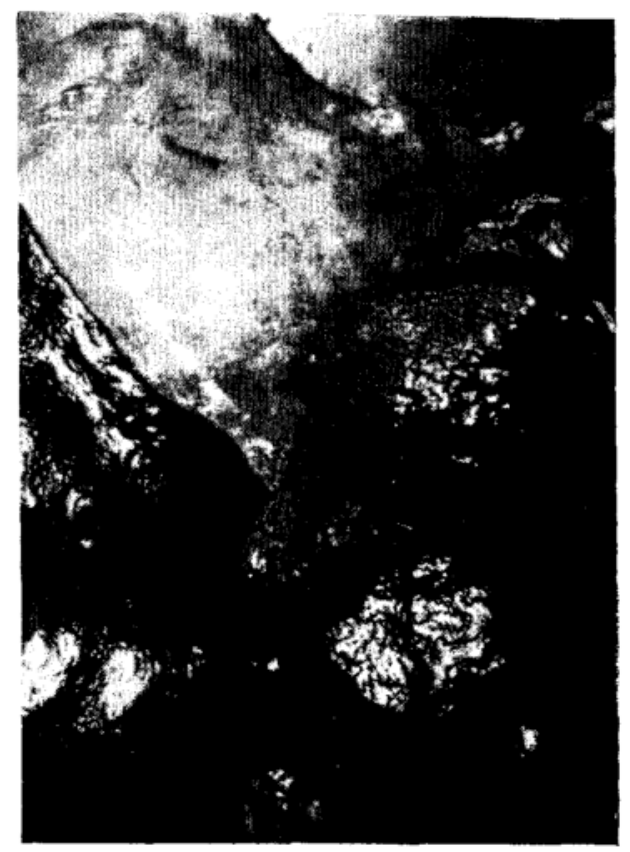

Figura 3 - Fotografia ilustrando pulmões e saco pericárdico. Pleuras lisas brilhantes e transparentes deixando ver tecido pulmonar congesto com áreas de hemorragia. 
Carvalho JEM, Marchiori ES, Silia JBG, Souza Netto BA, Tavares W, Paula AV. Comprometimento pulmonar na leptospirose. Revista da Sociedade Brasileira de Medicina Tropical 25:21-30, jan-mar, 1992.

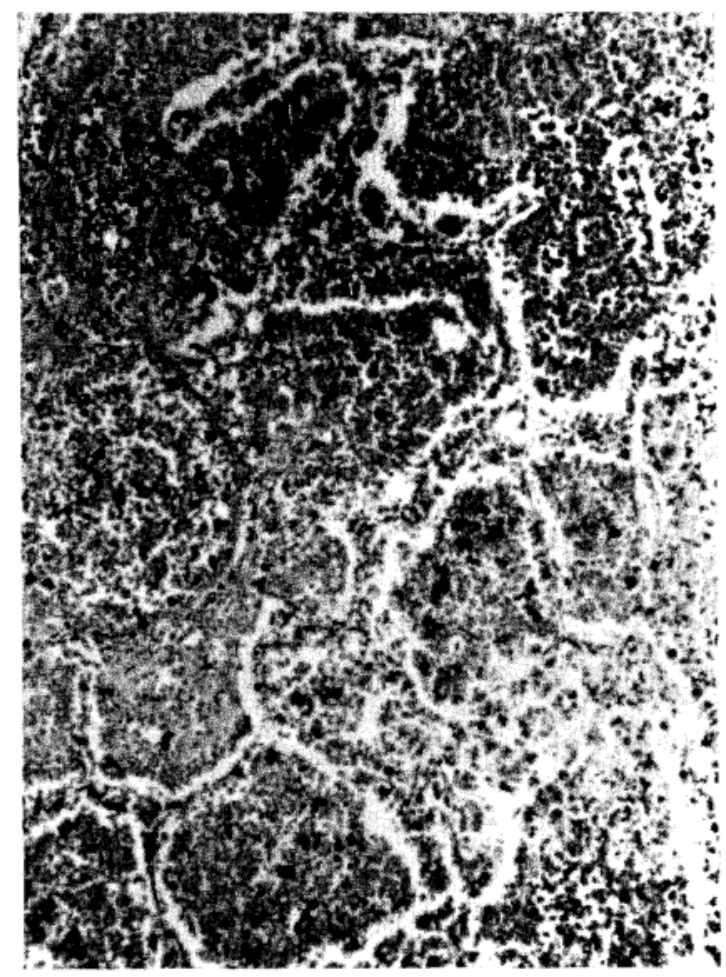

Figura 4 - Infiltração hemorrágica alveolar. Alvéolos preenchidos por hemácias em meio às quais observa-se abundante pimento de hemossiderina. (Hematoxilina-Eosina $100 \mathrm{X}$ ). arterial. Hipoxemia foi um achado freqüente $(60 \%$ dos casos), estando provavelmente relacionada aos importantes distúrbios que ocorrem ao nível alvéolocapilar, com conseqüente aumento do gradiente alveolar-arterial de oxigênio. Estes achados foram, também, descritos por Nery e cols ${ }^{21}$. O estudo radiológico do tórax em nossos pacientes revelou o predomínio do padrão de comprometimento alveolar, encontrado em 9 (60\%) dos casos, o que traduz a ocorrência de alveolite ${ }^{161722}$.

No estudo anatomopatológico que realizamos, macroscopicamente, a maioria dos nossos pacientes apresentava, nos pulmões, áreas vinhosas difusas, compatíveis com hemorragias generalizadas. $\mathrm{O}$ derrame pleural bilateral, encontrado em dois casos, sendo em um hemorrágico, apesar de raro, já foi descrito por outros autores ${ }^{3} 27$. Histologicamente, hemorragia, edema e congestão foram encontrados em $100 \%$ dos nossos casos, traduzindo-se clinicamente por dispnéia, hemoptise, tosse e, eventualmente, cianose. Estes aspectos foram também encontrados por Silverstein ${ }^{28}$, que refere ter encontrado hemorragia extensa em casos avançados, enfatizando que o comprometimento pulmonar na leptospirose consiste basicamente de uma pneumopatia hemorrágica. Entre nós, Pereira da Silva cols ${ }^{24}$, estudando a necrópsia de setepacientes

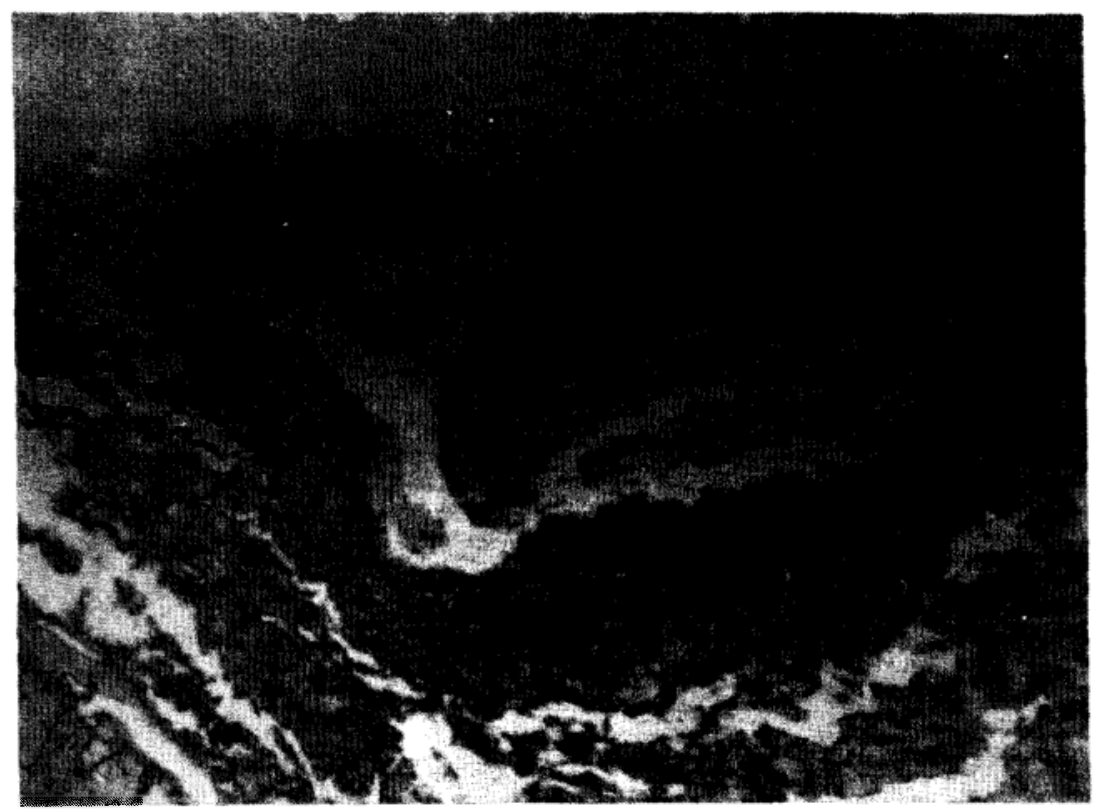

Figura 5 - Vaso pulmonar de pequeno calibre apresentando em sıa luz fornação trombótica onde se notan agregados de fibrina. (Hematoxilina-Fosfotungstica de Mallory $700 \mathrm{X})$. 
Carvalho JEM, Marchiori ES, Silva JBG, Souza Netto BA, Tavares W, Paula AV. Comprometimento pulmonar na leptospirose. Revista da Sociedade Brasileira de Medicina Tropical 25:21-30, jan-mar, 1992.

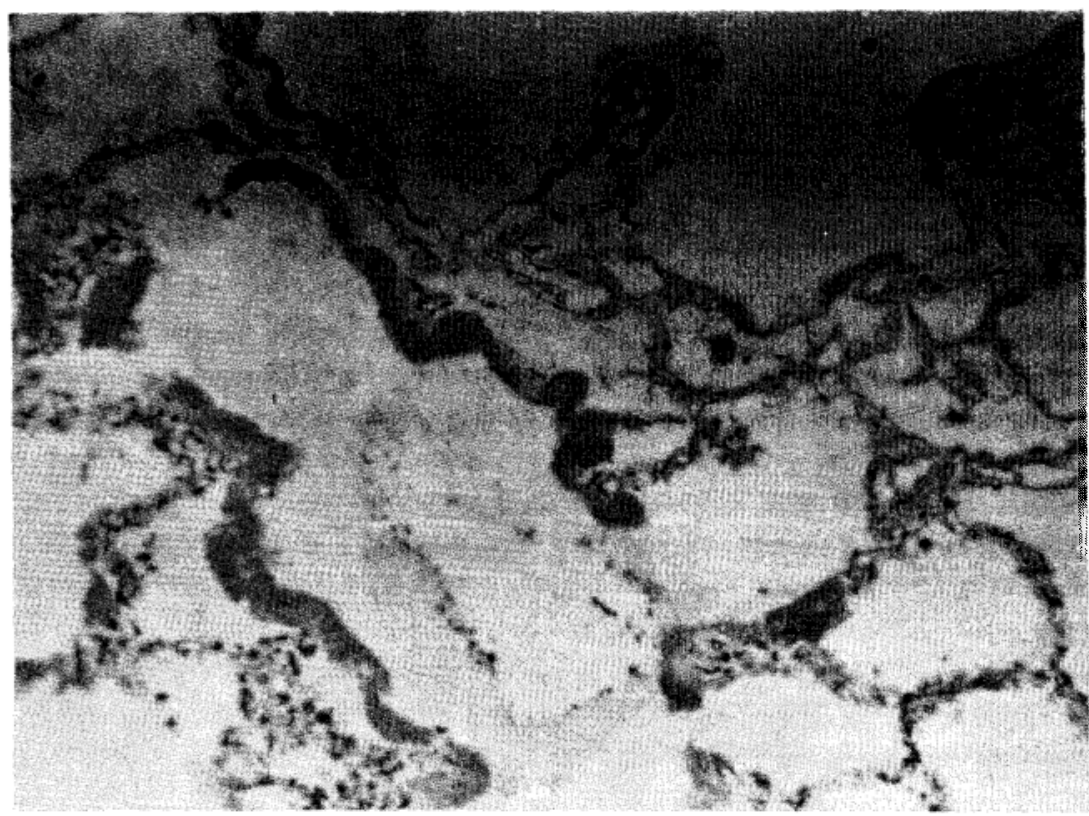

Figura 6 -Alvéolos contendo líquido de edema paredes alveolares atapetada por membranas hialinas. Septos alveolares apresentando discreto a moderado infiltrado inflamatório mononuclear. (Hematoxilina-Eosina $100 \mathrm{X}$ ).

com leptospirose com alterações pulmonares, e Ramachandran e Pereira ${ }^{27}$, estudando outros sete pacientes, constataram, respectivamente, hemorragia intra-alveolar em $100 \%$ e $70 \%$ dos casos.

Em nossa casuística, as hemoptises, geralmente de pequeno volume, apareceram precocemente, desaparecendo até o sétimo dia da doença, o que é relatado por outros autores ${ }^{24} 25$. Ocasionalmente, hemoptises maciças ocorrem, causando graves problemas anóxicos. Importante fato é que a ausência de hemoptises não é indicativo de menor extensão das lesões pulmonares; entretanto, em geral, pacientes com hemoptises mostram maior extensão das lesões pulmonares do que os sem hemoptise 2631 .

A patogênese da hemorragia pulmonar ainda permanece obscura. Segundo Carré e cols ${ }^{8}$, algumas hipóteses têm sido levantadas:

1. Um efeito citopatológico direto das leptospiras sobre o pulmão. A favor desta teoria está a precocidade do aparecimento das lesões, ainda na fase septicêmica;

2. Intervenção das toxinas liberadas pela lise das leptospiras, provocando dilatação e aumento da fragilidade dos capilares pulmonares;

3. Um mecanismo auto-imune, semelhante à síndrome de Goodpasture, com elevados níveis circulantes de anticorpos antimembrana basal, e consumo de complemento sérico total e da fração C3.

Recentemente, Brito e cols ${ }^{6}$, em trabalho experimental, usando culturas de leptospiras interrogans serovar ictero-haemorrhagiae, produziramem cobaias lesões em endotélio e epitélio, com ruptura de capilares e conseqüentemente hemorragia nos pulmões. Fragmentos semelhantes a leptospiras foram menos freqüentemente visualizados nos espaços alveolares, em microscopia eletrônica. Parece, assim, que fatores tóxicos do agente agressor, provavelmente componentes lipídicos e/ou glicolipoproteínas, contribuem para a patogênese das lesões hemorrágicas na leptospirose ${ }^{30}$. Deve-se, ainda, considerar que alteraçôes no mecanismo de coagulação do sangue podem contribuir para o surgimento da hemorragia pulmonar ${ }^{20}$, fato esteencontrado em nossa casuística.

Ainda sobre os achados histopatológicos, 0 infiltrado inflamatório mononuclear foi encontrado em apenas três (23\%) dos casos que estudamos, caracterizando o quadro de pneumonite. Tal alteração pode ser conseqüente à ação da leptospira sobre o parênquima pulmonar, aventando-se, também, a hipótese de um mecanismo imuno-alérgico semelhante àquele da síndrome de Loeffler ${ }^{23}$. Eventualmente, pacientes com leptospirose 
Carvalho JEM, Marchiori ES, Silva JBG, Souza Netto BA, Tavares W, Paula AV. Comprometimento pulmonar na leptospirose. Revista da Sociedade Brasileira de Medicina Tropical 25:21-30, jan-mar, 1992.

apresentam focos pneumônicos relacionados com infecções piogênicas secundárias ${ }^{23}$, fato que documentamos em três $(23 \%)$ dos nossos casos submetidos ao estudo anatomopatológico, traduzindo-se por broncopneumonia. Em um dos nossos pacientes havia infiltrado abundante, associado a discretas membranas hialinas, sugestivo de pneumonite urêmica, o que não é de se estranhar, pois ela faz parte da constelação mórbida da insuficiência renal grave. Em outros três pacientes, foi possível descrever a ocorrência da membrana hialina, expressão de importante agressão à membrana alvéolo-capilar, fazendo parte da síndrome de angústia respiratória do adulto (SARA). Em outros trabalhos 8112023 , houve também referência à SARA na leptospirose. Zaltman e cols $^{32}$ descrevem um caso de SARA em paciente acometido de leptospirose por serovar canicola que evoluiu para o óbito. Observaram à necrópsia infiltrado inflamatório associado a membranas hialinas e ruptura de capilares resultando em hemorragia alveolar, semelhante ao encontrado em três de nossos pacientes.

Importante, e até surpreendente, foram os achados indicativos de coagulação intravascular disseminada (CID), encontrada em $62 \%$ das necrópsias. No pulmão, particularmente, havia trombos de fibrina em $46 \%$ dos casos. Esses achados devem ser considerados, pois são raramente descritos na leptospirose $e^{4101829}$. Macedo e cols ${ }^{18}$ relataram um caso de leptospirose em que foi diagnosticada a CID, levando a um sangramento digestivo incontrolável. $O$ controle clínico e heparinização resultaram em cura do paciente. Por outro lado Pereira da Silva ${ }^{23}$ examinando a histopatologia em material proveniente de 10 necrópsias e 41 biópsias dos músculos gastrocnêmios não observou depósitos de fibrina em luz vascular. Nos três pacientes com SARA, foram observadas evidências de coagulação intravascular nos pulmões e em outros órgãos. Isto parece adquirir importância, já que em trabalhos realizados por Bone e $\operatorname{col}^{5}$ e Colman e Rubin ${ }^{9}$ foi verificado que pacientes com SARA e CID apresentavam maior hipoxemia e menor complacência pulmonar quando comparados a pacientes com SARA sem CID. Se o significado dessa associação, como causa ou efeito ainda permanece obscuro, o fato é que ela existe ${ }^{5}$, e tanto a SARA quanto a CID aparecem em pacientes com leptospirose.

Da análise dos dados apresentados, nasce a conclusão de se pesquisar sistematicamente a participação pulmonar no conjunto mórbido da leptospirose. Quer as lesões pulmonares dominem a cena, quer sejam epifenômeno no contexto patológico, o fato é que contribuem para a marcha da doença. É possível, ainda, que o domínio da complicação pulmonar possa, em certos casos, ser decisiva para a recuperação do paciente ${ }^{7} .0$ acurado exame clínico do aparelho respiratório acompanhado de exame radiológico seriado do tórax, e dosagem gasométrica do sangue arterial, são fatores fundamentais para o diagnóstico e orientação terapêutica do comprometimento pulmonar da leptospirose.

\section{SUMMARY}

To study the pulmonary complications in leptospirosis case records of 23 such patients admitted at the Hospital Universitário Antônio Pedro, Universidade Federal Fluminense, Niterói, Brasil, were reviewed. Hemoptysis were seen in $21.7 \%$ and sputal blood in $30.4 \%$ of patients. Arterial gasometry detected hypoxemia and hypocapnia in most cases. Thoracic radiology showed an alveolar pattern in $60 \%$ of the patients, alveolo-interstitial in $20 \%$, interstitial in $6 \%$, and in $14 \%$ the lungs were considered to be normal. Necropsy of 13 cases showed edema, congestion and hemorrhage in the lungs in all cases. Hyaline membrane was found in 30\% and fibrin thrombi in $46 \%$ of these cases, resulting in a diagnosis of adult respiratory distress syndrome and acute disseminated intravascular coagulation (consumption coagulopathy) in leptospirosis.

Key-words: Leptospirosis. Lung. Consumption coagulopathy. ARDS. Adult respiratory distress syndrome.

\section{REFERÊNCIAS BIBLIOGRÁFICAS}

1. Alexander AD. Leptospira. In: Lennete EH, Balous A, HauslerJunior WS, Shadomy HS (eds) Manual of clinical microbiology, $4^{\text {th }}$ editions, American Society for Microbiology, Washington p.473-478, 1985. 
Carvalho JEM; Marchiori ES, Silva JBG, Souza Netto BAS, Tavares W, Paula AV. Comprometimento pulmonar na leptospirose. Revista da Sociedade Brasileira de Medicina Tropical 25:21-30, jan-mar, 1992.

2. Almeida FSBM. Pulmão na Leptospirose. Revista Divisão Nacional Tuberculose 21:427-433, 1977.

3. Arean VM. The pathologic anatomy and pathogenesis of fatal human leptospirosis (Weil's disease). American Journal Pathology 40:293-423, 1962.

4. Ayrosa Galvão PA. Leptospiroses humanas. ARS Curandi 4:82-90, 1971.

5. Bone RC, Francis PB, Pierce AlC. Intravascular coagulation associated with the adult respiratory distress syndrome. American Journal of Medicine 61:585-589, 1976.

6. Brito T, Böhm GM, Yasuda PH. Vascular damage in acute eynerimental lentofnirncee of the Suines. pig. Journal Pathology 128:177-182, 1979.

7. Burke BJ, Jearle JF, Mattingly D. Leptospirosis presenting with profuse haemoptysis. Bristish Medical Journal 2:982, 1976.

8. Carré PH, Awia-Berod C, Duval G, Michault A. Manifestations pulmonaires predominantes au cours des leptospiroses ictero-hemorragiques. Révue Maladie Respiratoire 2:343-349, 1985.

9. Colman RN, Rubin RN. Disseminated intravascular coagulation: Etiologies, diagnosis and therapy. Infections in Medicine 1:23-28, 1986.

10. Comby F, Gauthier R, Naziwoff $O$. Nouvelle contribution a l'étude des leptospiroses a la reunion II. Anatomopathologie de dix cas mortels. Bulletin Société Pathologie Exotique 1:92-101, 1969.

11. Duval G, Michault A, Corsin JC, Awin-Berod C, Carré P, Geniu R. Syndrome de détresse respiratoire aigué revelateur d'une leptospirose icterohemorragique deux observations. La Presse Medicale 14:167-172, 1985.

12. Edwards GA, Domm BM. Human leptospirosis. Medicine 39:117-156, 1960.

13. Farrar WE L eptospira species (Leptospirasis, ln: Mandell GL (ed) Principles and practice of infectious diseases. 2edition, John Wiley \& Sons, New York p.1338-1343, 1985.

14. Feigin RD, Anderson DE. Human leptospirosis. CRC Critical Reviews in Clinical Laboratory Sciences 5:413-467, 1975.

15. Inada $R$, Ido $Y$. The etiology, mode of infection and specific therapy of Weil's disease spirochaetosis icterohemorragica. Journal Expect Medicine 23:397, 1916.

16. Keogh BA, Crystal RG. Alveolitis: the key to the interstitial lung desordens. Thorax 37:1-10, 1982.

17. Liebow AA, Carrington $\mathrm{CB}$. The Interstitial pneumonias. In: Simon M (ed) Frontiers of pulmonary radiology. Grune \& Stratton, New York, 1969.

18: Macedo V, Figueiredo JFM, Carvalho E, Barbosa E. Coagulação intravascular disseminada na leptospirose. Revista de Patologia Tropical 3:363366, 1973

19. Martin L, Pettit A. Spirochetose icterohémorragique. In: Monografias do Institute Pasteur, Libraries de L'Académie Médicine, Masson et Cience, Paris p.1-11, 1919.

20. Miller NG, Allen JE, Wilson RB. The pathogenesis of hemorrhage in the lung of the hamster during

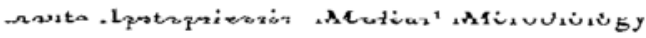
Immunology 160:269-278, 1974.

21. Nery LE, Paula AB, Nakatani J, Santos ML, Ratto OR. Clinical radiological an functional pulmonary manifestation in patients with leptospirosis. Revista do Instituto de Medicina Tropical de São Paulo 19:366-373, 1977.

22. Paula A. Da tisiologia à pneumologia. Uma evolução em meio século. Jornal Brasileiro de Medicina 46:41-68, 1984

23. Pereira da Silva JJ. Formas graves de leptospirose - contribuição ao seu estudo clínico. Tese de Mestrado, Universidade Federal do Rio de Janeiro, Rio de Janeiro, 1974.

24. Pereira da Silva JJ, Paiva LM, Silva JBG, Alves Netto B. Estudo do Comprometimento pulmonar na doença de Well. Revista do Instituto de Medicina Tropical de São Paulo 18:387-392, 1976.

25. Petricevic I, Tomljenovic V. Milijarne pluene promjene u toku leptospiroze prikaz bolesnika. Lijecnicki Vjesnik 102:135-136, 1980.

26. Poh SC, Soh CS. Lung manifestations in leptospirosis. Thorax 25:751-755, 1970.

27. Ramechandran S, Poroira MVIF. Cardias arrut pulmonary involvement in leptospirosis. Transactions of the Royal Society of Tropical Medicine and Hygiene 71:56-59, 1977.

28. Silverstein CM. Pulmonary manifestation of leptospirosis. Radiology 61:327-334, 1953.

29. Sitprija V, Pipatanagul V, Mertowidjojo K, Boonpucknavig V, Boonpucknavig S. Pathogenesis of renal disease in leptospirosis: clinical and experimental studies. Kidney Internal 17:827-836, 1980.

30. Vinh T, Adler B, Fayne S. Glycoliprotein cytotoxin from Leptospira Interrogans serovar copenhageni. Journal General Microbiology 132:111-123, 1986. 
Carvalho JEM; Marchiori ES, Silva JBG, Souza Netto BAS, Tavares W, Paula AV. Comprometimento pulmonar na leptospirose. Revista da Sociedade Brasileira de Medicina Tropical 25:21-30, jan-mar, 1992.

2. Almeida FSBM. Pulmão na Leptospirose. Revista Divisão Nacional Tuberculose 21:427-433, 1977.

3. Arean VM. The pathologic anatomy and pathogenesis of fatal human leptospirosis (Weil's disease). American Journal Pathology 40:293-423, 1962.

4. Ayrosa Galvão PA. Leptospiroses humanas. ARS Curandi 4:82-90, 1971.

5. Bone RC, Francis PB, Pierce AlC. Intravascular coagulation associated with the adult respiratory distress syndrome. American Journal of Medicine 61:585-589, 1976.

6. Brito T, Böhm GM, Yasuda PH. Vascular damage in acute experimental leptospiroses of the Guineapig. Journal Pathology 128:177-182, 1979.

7. Burke BJ, Jearle JF, Mattingly D. Leptospirosis presenting with profuse haemoptysis. Bristish Medical Journal 2:982, 1976.

8. Carré PH, Awia-Berod C, Duval G, Michault A. Manifestations pulmonaires predominantes au cours des leptospiroses ictero-hemorragiques. Révue Maladie Respiratoire 2:343-349, 1985.

9. Colman RN, Rubin RN. Disseminated intravascular coagulation: Etiologies, diagnosis and therapy. Infections in Medicine 1:23-28, 1986.

10. Comby F, Gauthier R, Naziwoff $O$. Nouvelle contribution a l'étude des leptospiroses a la reunion II. Anatomopathologie de dix cas mortels. Bulletin Société Pathologie Exotique 1:92-101, 1969.

11. Duval G, Michault A, Corsin JC, Awin-Berod C, Carré $P$, Geniu R. Syndrome de détresse respiratoire aigué revelateur d'une leptospirose icterohemorragique deux observations. La Presse Medicale 14:167-172, 1985.

12. Edwards GA, Domm BM. Human leptospirosis. Medicine 39:117-156, 1960.

13. Farrar WE. Leptospira species (Leptospirosis) In: Mandell GL (ed) Principles and practice of infectious diseases. 2edition, John Wiley \& Sons, New York p.1338-1343, 1985.

14. Feigin RD, Anderson DE. Human leptospirosis. CRCCritical Reviews in Clinical Laboratory Sciences 5:413-467, 1975.

15. Inada R, Ido $Y$. The etiology, mode of infection and specific therapy of Weil's disease spirochaetosis icterohemorragica. Journal Expect Medicine 23:397, 1916.

16. Keogh BA, Crystal RG. Alveolitis: the key to the interstitial lung desordens. Thorax 37:1-10, 1982.

17. Licbow AA, Carrington $\mathrm{CB}$. The Interstitial pneumonias. In: Simon M (ed) Frontiers of pulmonary radiology. Grune \& Stratton, New York, 1969.

18: Macedo V, Figueiredo JFM, Carvalho E, Barbosa E. Coagulação intravascular disseminada na leptospirose. Revista de Patologia Tropical 3:363366, 1973.

19. Martin L, Pettit A. Spirochetose icterohémorragique. In: Monografias do Institute Pasteur, Libraries de L'Académie Médicine, Masson et Cience, Paris p.1-11, 1919.

20. Miller NG, Allen JE, Wilson RB. The pathogenesis of hemorrhage in the lung of the hamster during acute leptospirosis. Medical Microbiology Immunology 160:269-278, 1974.

21. Nery LE, Paula AB, Nakatani J, Santos ML, Ratto OR. Clinical radiological an functional pulmonary manifestation in patients with leptospirosis. Revista do Instituto de Medicina Tropical de São Paulo 19:366-373, 1977.

22. Paula $\mathrm{A}$. Da tisiologia à pneumologia. Uma evolução em meio século. Jornal Brasileiro de Medicina 46:41-68, 1984.

23. Pereira da Silva JJ. Formas graves de leptospirose - contribuição ao seu estudo clínico. Tese de Mestrado, Universidade Federal do Rio de Janeiro, Rio de Janeiro, 1974.

24. Pereira da Silva JJ, Paiva LM, Silva JBG, Alves Netto B. Estudo do Comprometimento pulmonar na doença de Well. Revista do Instituto de Medicina Tropical de São Paulo 18:387-392, 1976.

25. Petricevic I, Tomljenovic V. Milijarne plucne promjene u toku leptospiroze prikaz bolesnika. Lijecnicki Vjesnik 102:135-136, 1980.

26. Poh SC, Soh CS. Lung manifestations in leptospirosis. Thorax 25:751-755, 1970.

27. Ramachandran S, Pereira MVF. Cardiac and pulmonary involvement in leptospirosis. Transactions of the Royal Society of Tropical Medicine and Hygiene 71:56-59, 1977.

28. Silverstein CM. Pulmonary manifestation of leptospirosis. Radiology 61:327-334, 1953.

29. Sitprija V, Pipatanagul V, Mertowidjojo K, Boonpucknavig V, Boonpucknavig S. Pathogenesis of renal disease in leptospirosis: clinical and experimental studies. Kidney Internal 17:827-836, 1980.

30. Vinh T, Adler B, Fayne S. Glycoliprotein cytotoxin from Leptospira Interrogans serovar copenhageni. Journal General Microbiology 132:111-123, 1986. 
Carvalho JEM, Marchiori ES, Silva JBG, Souza Netto BAS, Tavares W, Paula AV. Comprometimento pulmonar na leptospirose. Revista da Sociedade Brasileira de Medicina Tropical 25:21-30, jan-mar, 1992.

31. Wang CN, Liu J, Chang TF, Cheng WJ, Wo MY, Hung AT. Studies on anicteric leptospirosis III Roent Genelogic observations of pulmonary changes. Chinese Medical Journal 84:298-306, 1965.
32. Zaltzman M, Kallenbach JM, Goss GO, Lewis M, Zwi S, Gear JHS. Adult respiratory distress syndrome in leptospira canicola infection. British Medical Journal 283:519-520, 1981. 